Supporting Information

\title{
Two-Pathway Viewpoint to Interpret Quantum Interference in Molecules Containing Five-Membered Heterocycles: Thienoacenes as Examples
}

Yang Li†, Xi Yuł, Yonggang Zhen†, Huanli Dongł*, Wenping Hu†ł*

$\dagger$ †eijing National Laboratory for Molecular Science, Key Laboratory of Organic

Solids, Institute of Chemistry, Chinese Academy of Sciences, Beijing 100190, China

¥Tianjin Key Laboratory of Molecular Optoelectronic Sciences, Department of Chemistry, School of Science, Tianjin University and Collaborative Innovation Center of Chemical Science and Engineering, Tianjin 300072, China 


\section{Content:}

1. Verification of the TB parameters

2. Comparison of $\left|G^{m o l}(E)\right|^{2}$ with the corresponding $\left|G^{C}(E)\right|^{2}$

3. Characteristics of the zeroth Green functions of acenes

4. Derivation of the segmented analysis

5. Intra-segmental and inter-segmental Green functions

6. Atomic projected density of states (PDOS) analysis

\section{Verification of the TB parameters}

Zeroth Green functions calculated under the TB approach are compared with DFT results using SZ basis set. In DFT calculations, the zeroth Green functions are obtained by $G(E)=(E S-H)^{-1}$ where $H$ and $S$ are the DFT Hamiltonians and overlap matrices. We assume the anchor groups only interact with the $p_{z}$ orbitals of the connecting atoms here. For a metal-molecule-metal junction, the equilibrium Fermi level of the electrode tends to lie in between the HOMO and LUMO resonances of the molecule. ${ }^{1-3}$ As a result, in this section we will choose the zero energy of the DFT results as the meaningless Fermi level of the molecule situated in a big cell with at least $14 \AA$ of vacuum in all three rectangular directions when the electronic temperature is $300 \mathrm{~K}$. Zero energy under the TB approach is set as the on-site energy of C atoms.

Molecules studied in this work are illustrated in Scheme S1. Typical thienoacenes with acene edges (DBT, BTBT-1, BTBT-2), thiophene edges (BDT-1, BDT-2) combined with thiophene (T) and dithiophenes (DT-1, DT-2) are chosen to verify our TB parameters. We draw the modulus square of some zeroth Green functions calculated under the TB approach along with the corresponding DFT results in Figure $\mathrm{S} 1$ and $\mathrm{S} 2$ where $\mathrm{X}(i-j)$ denotes the connection between atom $i$ and $j$ in molecule X. According to these results, although the HOMO-LUMO gap under the TB approach may not be exact, the $|G(E)|^{2}$ curves between HOMO and LUMO resonances are in good accordance with DFT results. Thus we have confirmed that the set of TB parameters are suitable for calculating the molecular Green functions, 
although in five-membered heterocycles the on-site energies and hopping integrals of the $\mathrm{C}$ backbone are not identical ${ }^{4}$.

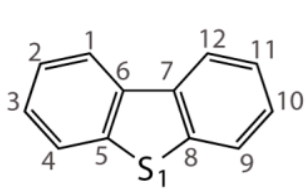

DBT

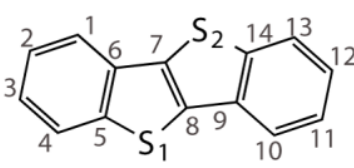

BTBT-1

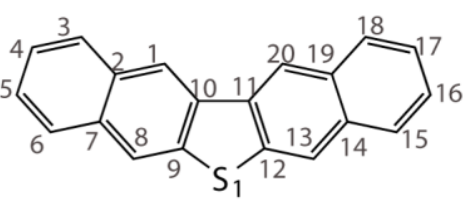

DNT

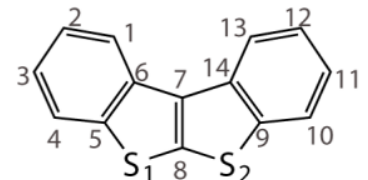

BTBT-2<smiles>c1cc2cc3sccc3cc2s1</smiles>

BDT-1<smiles>c1cc[se]c1</smiles>

$\mathrm{T}$<smiles>c1cc2cc3ccsc3cc2s1</smiles>

BDT-2

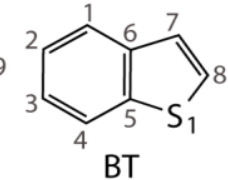

BT<smiles>c1cc2ccsc2s1</smiles>

DT-2<smiles>c1ccc2c(c1)sc1c2sc2c3ccccc3sc21</smiles>

DBTDT-1<smiles>c1cc2sccc2s1</smiles>

DT-1<smiles></smiles>

DBTDT-2

Scheme S1. Representative fused thienoacenes along with the atom indexes.
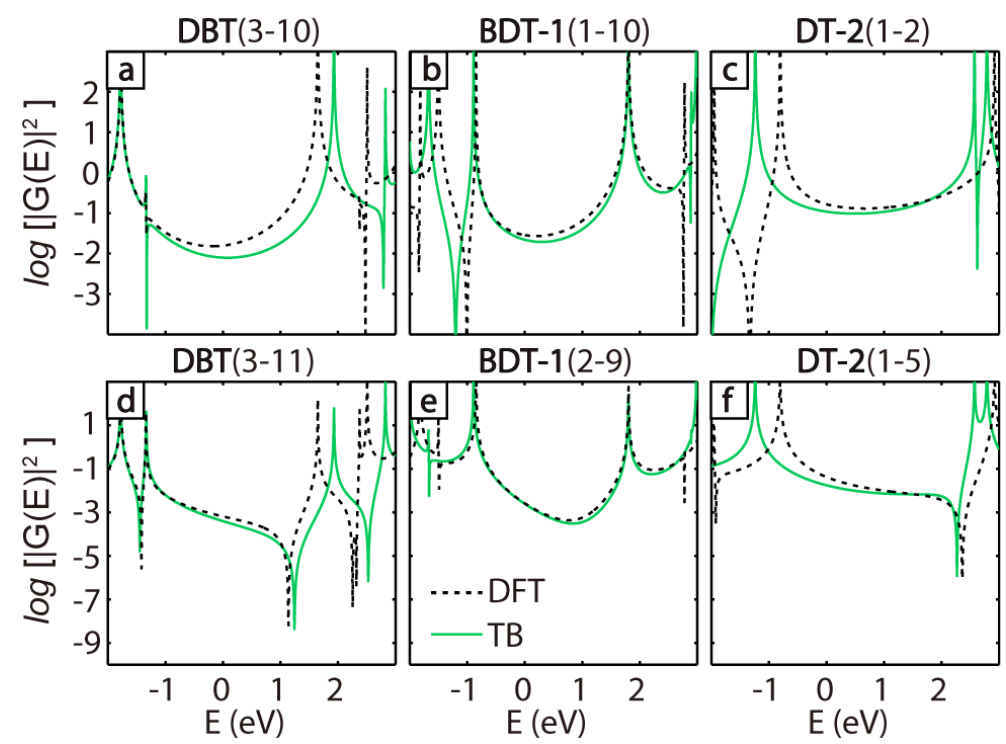

Figure S1. $\left|G^{\text {mol }}(E)\right|^{2}$ calculated under the TB approach (green solid lines) compared with those calculated by DFT under SZ basis set (black dashed lines) for several connections in different thienoacenes. 

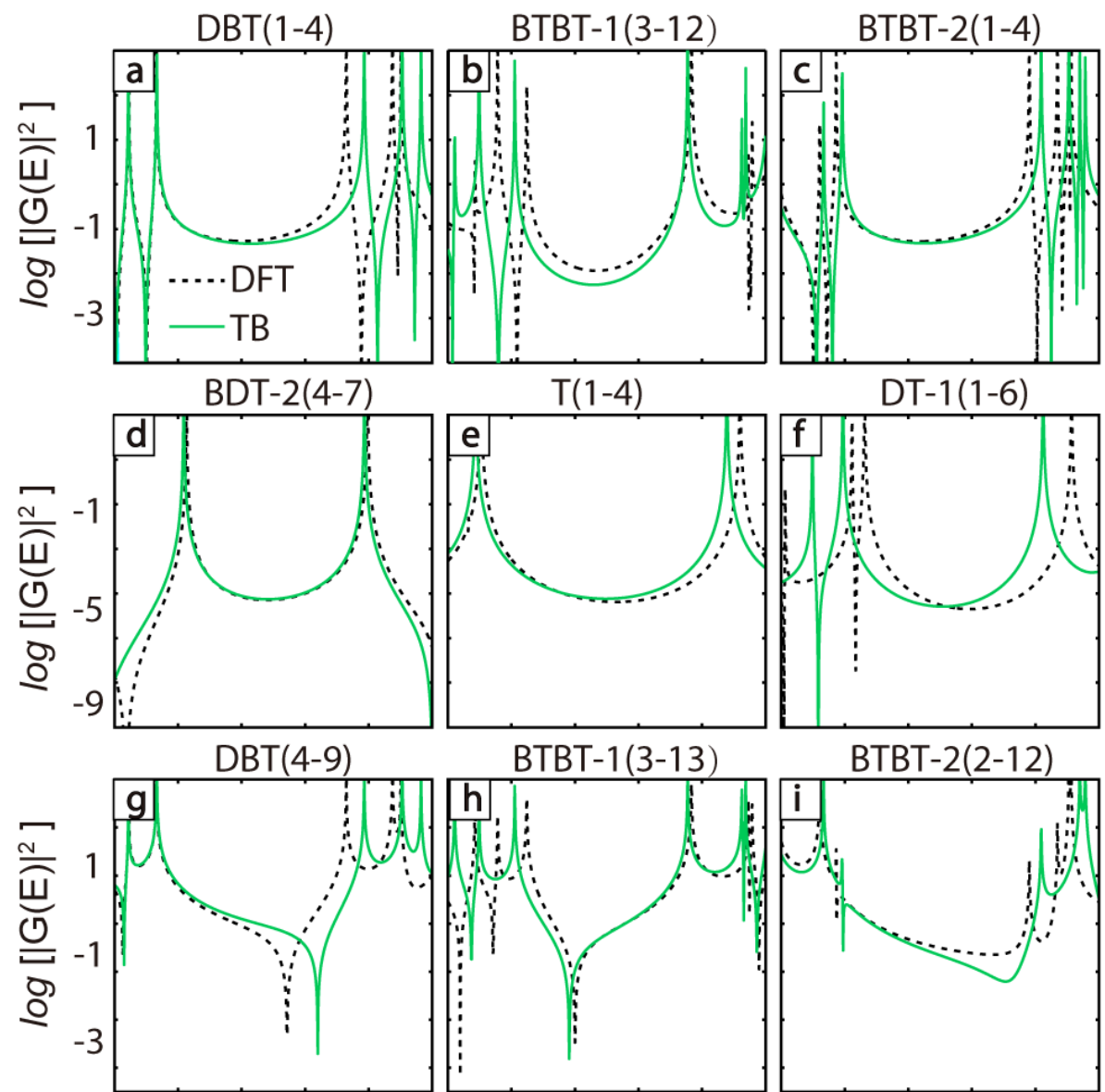

BTBT-2(2-12)
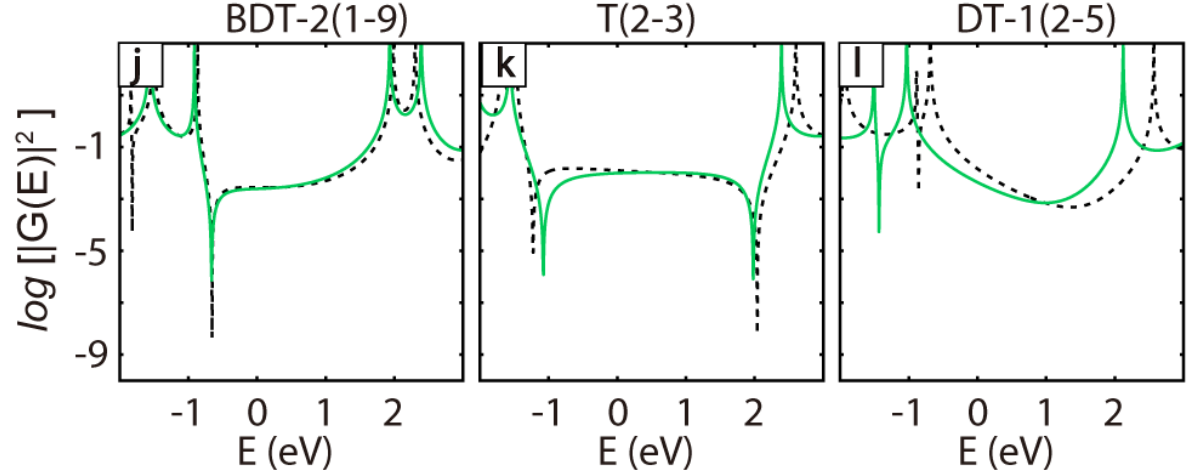

Figure S2. More $\left|G^{m o l}(E)\right|^{2}$ calculated under the TB approach (green solid lines) compared with those calculated by DFT under SZ basis set (black dashed lines). 
2. Comparison of $\left|G^{m o l}(E)\right|^{2}$ with the corresponding $\left|G^{C}(E)\right|^{2}$
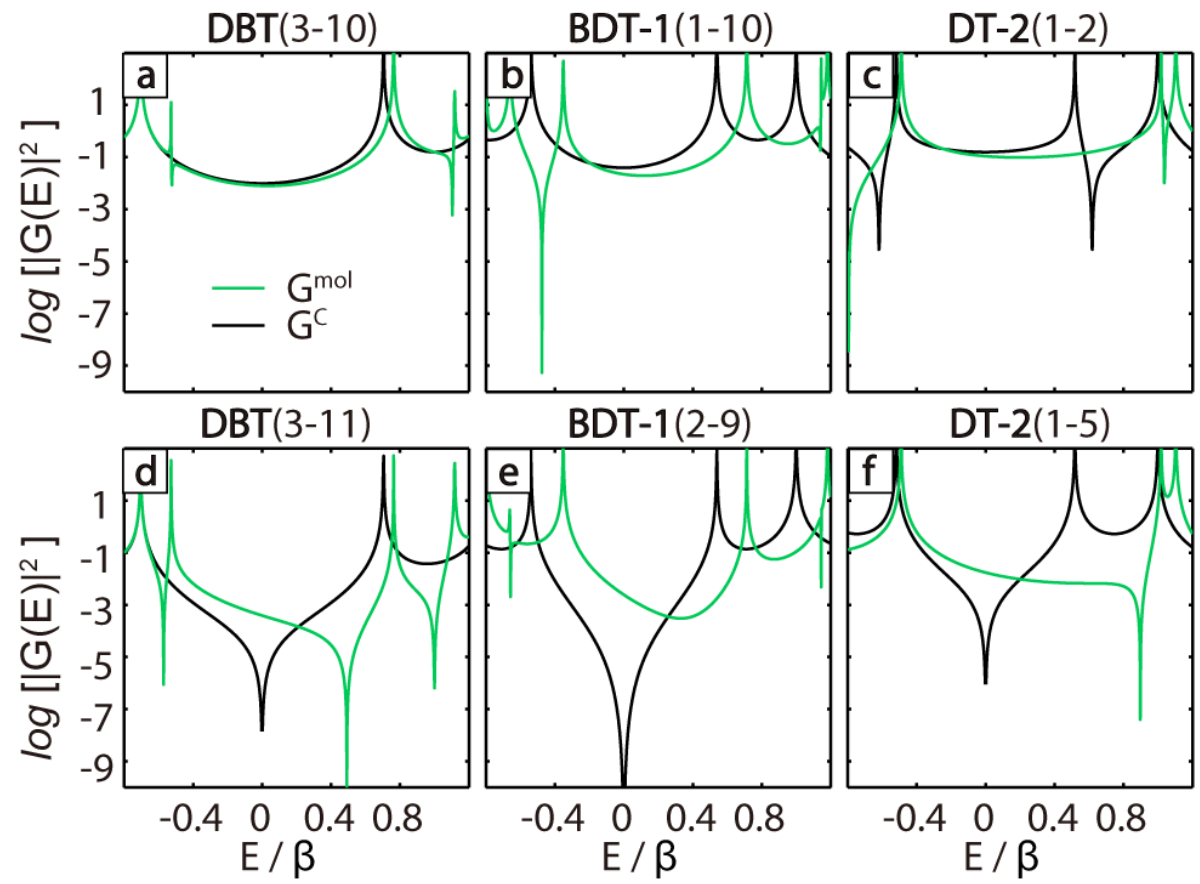

Figure S3. $\left|G^{m o l}(E)\right|^{2}$ calculated under the TB approach (green) compared with the corresponding $\left|G^{C}(E)\right|^{2}$ (black). The C backbone exhibit CQI for (a-c) and DQI for (d-f). 

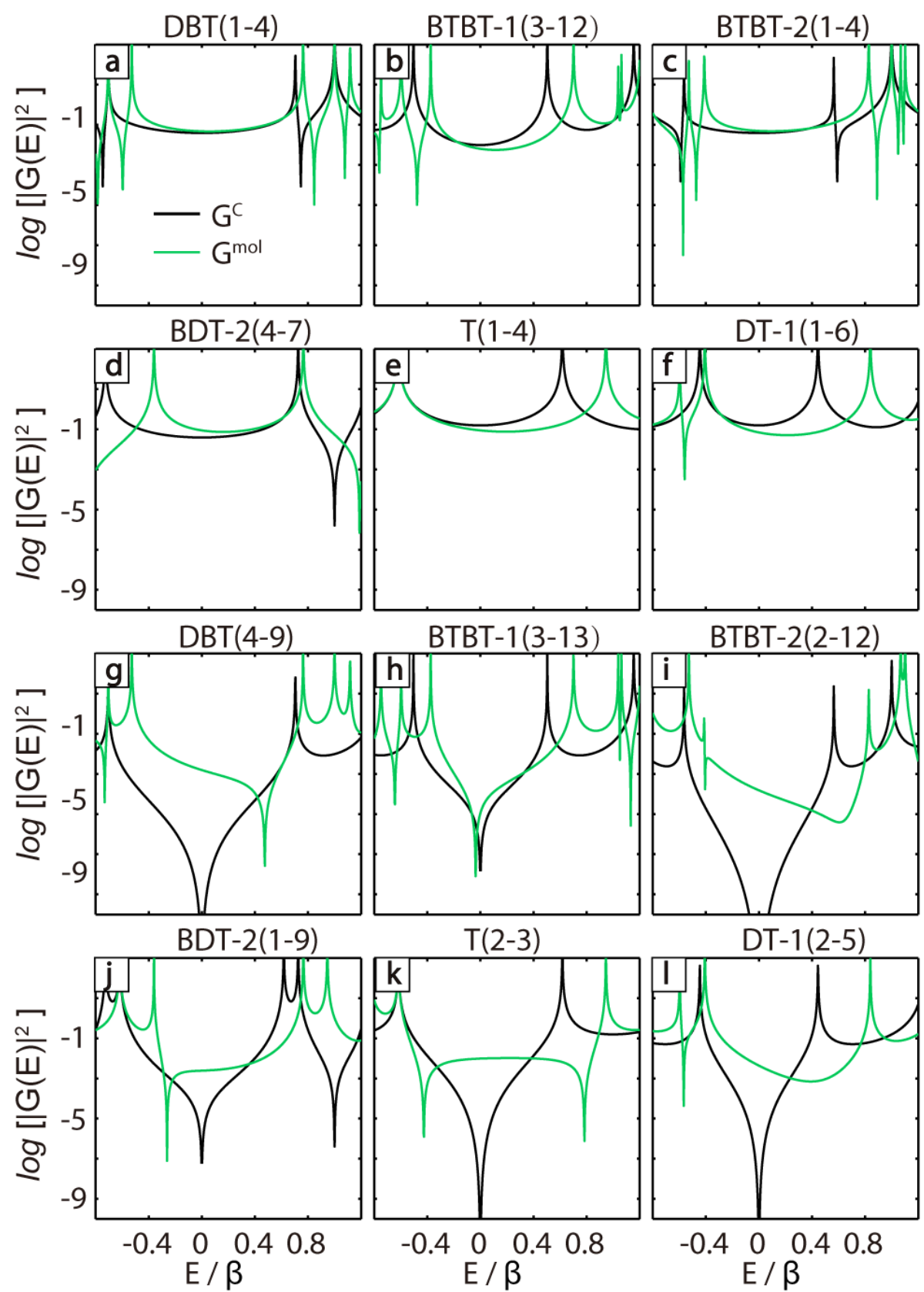

Figure S4. More $\left|G^{m o l}(E)\right|^{2}$ calculated under the TB approach (green) compared with the corresponding $\left|G^{C}(E)\right|^{2}$ (black). The C backbone exhibit CQI for (a-f) and DQI for $(\mathrm{g}-1)$ 


\section{Characteristics of the zeroth Green functions of acenes}

In common thienoacenes ${ }^{5}$, the segments are C-C pairs or acenes. Before performing the segmented analysis, some useful properties of the zeroth Green functions of the segments will be pointed out in this section. Because of the alternant feature, we can rearrange the connection matrix by writing the odd atoms first. Thus the Hamiltonian takes the form ${ }^{6}$

$$
H=-\left(\begin{array}{cc}
O & C \\
C^{T} & O
\end{array}\right)
$$

Here $C$ is the connection matrix between starred and unstarred atoms. The TB parameter $\beta$ is used as the unit of energy and Hamiltonian for simplicity, so that the unit of Green functions is $1 / \beta$. Through direct matrix inversion, the Green function can be written as

$$
G(E)=\left(\begin{array}{cc}
E I & C \\
C^{\prime} & E I
\end{array}\right)^{-1}=\left(\begin{array}{cc}
-E\left(C C^{\prime}-E^{2} I\right)^{-1} & \left(C C^{\prime}-E^{2} I\right)^{-1} C \\
C^{\prime}\left(C C^{\prime}-E^{2} I\right)^{-1} & -E\left(C^{\prime} C-E^{2} I\right)^{-1}
\end{array}\right)
$$

Obviously, starred-unstarred connections (or odd-even connections before the rearrangement) lead to even Green functions, while starred-starred (odd-odd) or unstarred-unstarred (even-even) connections lead to odd Green functions which are always zero at $E=0$. The latter is the so-called easy zero leading to the destructive QI

effect of odd-odd and even-even connections in alternant molecules. ${ }^{7}$ Besides, if an even Green function coincides with zero at $E=0$ it is called a hard zero. This parity law holds in both the whole $\mathrm{C}$ backbone and the segments of thienoacenes. We draw the zeroth Green functions of $\mathrm{C}-\mathrm{C}$ pair and benzene in Figure 2c, and naphthalene in Figure S5 as examples.

Furthermore, we concentrate on acenes shown in Figure S6. Because of the parity feature, at zero energy only odd-even connections may lead to non-zero Green functions. Without loss of generality we use $i$ to indicate an odd number and $j$ to indicate an even number. For an acene containing $N$ rings shown in the frame, three regular rules can be verified for $N=1$ and $N=2$ where the zeroth Green function at $E$ $=0$ is defined as $G^{N}$ : 


$$
\left\{\begin{array}{l}
G_{12}^{N}=\frac{N}{N+1} \in(0,1), G_{23}^{N}=\frac{1}{N+1} \in(0,1) \\
\left|G_{1 j / 3 j}^{N}\right| \in(0,1),\left|G_{2 i / 4 i}^{N}\right| \in(0,1) \\
\left|\frac{G_{i 2}^{N} G_{j 3}^{N}}{G_{i j}^{N}}\right| \leq \frac{N}{N+1},\left|\frac{G_{i 2}^{N} G_{j 1}^{N}}{G_{i j}^{N}}\right| \leq \frac{N}{N+1}
\end{array}\right.
$$

Specicically we point out that for any acene, sites 1-4 include all the possible sites an acene with $N$ rings may share with a thiophene ring using symmetry.

Now mathematical induction will be used supposing Equation S3 holds for an acene containing $N$ rings. As Figure $\mathrm{S} 6$ we add a new ring to an existing acene. Initial sites 1-4 are indicated as 1'-4' instead. The zeroth Green function of the new structure at zero energy takes the form

$$
\begin{aligned}
G^{N+1} & =\left(\begin{array}{cc}
C^{\text {out }} & T \\
T^{T} & C^{N}
\end{array}\right)^{-1}=\left(\begin{array}{ccc}
G^{\text {out }} & G^{\text {out }- \text { in }} \\
G^{\text {in-out }} & G^{\text {in }}
\end{array}\right) \\
C^{\text {out }} & =\left(\begin{array}{llll}
0 & 1 & 0 & 0 \\
1 & 0 & 1 & 0 \\
0 & 1 & 0 & 1 \\
0 & 0 & 1 & 0
\end{array}\right), T=\left(\begin{array}{llllll}
0 & 1 & 0 & 0 & 0 & \ldots \\
0 & 0 & 0 & 0 & 0 & \ldots \\
0 & 0 & 0 & 0 & 0 & \ldots \\
0 & 0 & 1 & 0 & 0 & \ldots
\end{array}\right)
\end{aligned}
$$

The superscripts in and out indicate the sites inside and outside the frame. Through matrix inversion we firstly obtain

$$
G^{\text {out }}=\left[C^{\text {out }}-T G^{N} T^{T}\right]^{-1}=\left(\begin{array}{cccc}
0 & a_{N+1} & 0 & -a_{N+1} \\
a_{N+1} & 0 & 1-a_{N+1} & 0 \\
0 & 1-a_{N+1} & 0 & a_{N+1} \\
-a_{N+1} & 0 & a_{N+1} & 0
\end{array}\right)
$$

The value of $a_{N+1}$ is

$$
a_{N+1}=\frac{1}{1+G_{23}^{N}}
$$

As long as $G^{N}{ }_{23}>0, a_{N+1}$ and (1- $\left.a_{N+1}\right)$ corresponding to the ortho connections 1-2 and 2-3 are always in the range $(0,1)$. Taking advantage of the first line in Equation S3, the following equation is proved to hold for any value of $\mathrm{N}$.

$$
a_{N}=\frac{N}{N+1}
$$

At the same time the first line of Equation S3 is demonstrated. Moreover, in a C-C 
pair we have $G_{12}=1$ instead.

Secondly, for connections between sites outside the frame and inside the frame we have

$$
G^{\text {out }- \text { in }}=-G^{\text {out }} T G^{N}=\left(\begin{array}{lllll}
0 & a_{N+1} G_{32}^{N} & 0 & a_{N+1} G_{34}^{N} & \ldots \\
-a_{N+1} G_{21}^{N} & 0 & -a_{N+1} G_{23}^{N} & 0 & \ldots \\
0 & -a_{N+1} G_{32}^{N} & 0 & -a_{N+1} G_{34}^{N} & \ldots \\
a_{N+1} G_{21}^{N} & 0 & a_{N+1} G_{23}^{N} & 0 & \ldots
\end{array}\right)
$$

In other words

$$
\left\{\begin{array}{l}
G_{2 i}^{N}=-\frac{N}{N+1} G_{2 i}^{N-1}, G_{4 i}^{N}=\frac{N}{N+1} G_{2 i}^{N-1}, i=3,5,7, \ldots \\
G_{1 j}^{N}=\frac{N}{N+1} G_{3 j}^{N-1}, G_{3 j}^{N}=-\frac{N}{N+1} G_{3 j}^{N-1}, j=4,6,8, \ldots
\end{array}\right.
$$

The absolute values of these Green functions will not exceed 1 as long as those of $G^{N-1}{ }_{2 i}$ and $G^{N-1}{ }_{3 j}$ are less than 1 . Then the second line of Equation S3 is also demonstrated.

At last, Green functions of connections between sites inside the frame take the following form

$$
G^{i n}=G^{N}+G^{N} T^{T}\left(C^{N}-T G^{N} T^{T}\right)^{-1} T G^{N}
$$

From the above results we obtain

$$
G_{i j}^{i n}=G_{i^{\prime} j^{\prime}}^{N}-a_{N} G_{i^{\prime} 2}^{N} G_{j^{\prime} 3}^{N}
$$

Here $i^{\prime}=i-4$ and $j^{\prime}=j-4$ denote the indexes of the structure inside the frame. Below the third line of Equation S3 will be proved a little complicatedly. First we list the value of $G^{N+1}{ }_{i j}, G^{N+1}{ }_{i 2} \mathrm{G}^{N+1}{ }_{j 1}$ and $G^{N+1}{ }_{i 2} G^{N+1}{ }_{j 3}$ for all possible choices of even $i$ and odd $j$ in Table S1. Except for the last line, it is clear that the ratio $G^{N+1}{ }_{i 2} \mathrm{G}^{N+1}{ }_{j 1} / G^{N+1}$ ij is in the range $\left[-1+a_{N+1}, a_{N+1}\right]$ and $G^{N+1}{ }_{i 2} \mathrm{G}^{N+1}{ }_{j 3} / G^{N+1}{ }_{i j}$ is in the range [- $\left.a_{N+1}, 1-a_{N+1}\right]$. In fact, these restrictions are always true in benzene and naphthalene even if $i>4$ and $j>4$.

Suppose these restrictions hold when there are $N$ rings. Under the $\mathrm{i}>4$ and $\mathrm{j}>4$ condition we obtain

$$
\frac{G_{i 2}^{N+1} G_{j 3}^{N+1}}{G_{i j}^{N+1}}=\frac{a_{N+1}^{2}\left(G_{i^{\prime} 2}^{N} G_{j^{\prime} 3}^{N} / G_{i^{\prime} j^{\prime}}^{N}\right)}{1-a_{N+1}\left(G_{i^{\prime} 2}^{N} G_{j^{\prime} 3}^{N} / G_{i^{\prime} j^{\prime}}^{N}\right)}
$$


The function $f(x)=a^{2} x /(1-a x)$ is monotonically increasing due to its derivative $f^{\prime}(x)=$ $a^{2} /(1-a x)^{2}$ except for the singular point $x=1 / a$, which exceeds the range of $x$. Because the value of $G^{N}{ }_{i 2} \mathrm{G}^{N}{ }_{j 3} / G^{N}{ }_{i j}$ is in the range $\left[-a_{N}, 1-a_{N}\right]$, the following relation will always hold true:

$$
-a_{N+1}<-\frac{N}{2(N+2)} \leq \frac{G_{i 2}^{N+1} G_{j 3}^{N+1}}{G_{i j}^{N+1}} \leq \frac{1}{N+2}=1-a_{N+1}
$$

Hence mathematical induction can spread this relation to any value of N. On the other hand, the ratio $G^{N+1}{ }_{i 2} \mathrm{G}^{N+1}{ }_{j 1} / G^{N+1}{ }_{i j}$ is $-G^{N}{ }_{i 2} \mathrm{G}^{N}{ }_{j 3} / G^{N}{ }_{i j}$ when $\mathrm{i}>4$ and $\mathrm{j}>4$, so that it in the range $\left[-1+a_{N+1}, a_{N+1}\right)$. Totally for all the nine cases listed in Table S1, the absolute value of $\left|G^{N+1}{ }_{i 2} \mathrm{G}^{N+1}{ }_{11} / G^{N+1}{ }_{i j}\right|$ and $\left|G^{N+1}{ }_{i 2} \mathrm{G}^{N+1}{ }_{j 1} / G^{N+1}{ }_{i j}\right|$ will not exceed $a_{N+1}$ which is the same with the third line of Equation S3. For a C-C pair, a similar relation will become $G_{12} G_{21} / G_{12}=1$.

At the end of this section, we will restate Equation S3 in a different way. Suppose $g$ is the Green function of a C-C pair or an acene at $E=0$. It is certainly an alternant molecule, so that we can define odd sites starred and even sites unstarred. Unstarred site $p$ and starred site $m$ are adjacent sites on the "edge" of the segment which can be shared with a thiophene ring. In fact all the possible $m-p$ pairs are 1-2, 3-2 and 3-4 in Figure S6. Then the following four relations hold true:

(1) The Green function of the molecule exhibits even parity for starred-unstarred connections, and odd parity for starred-starred and unstarred-unstarred connections.

(2) The value of $g_{m p}$ is in the range $(0,1]$.

(3) The values of $\left|g_{i p}\right|$ and $\left|g_{j m}\right|$ are in the range $[0,1]$.

(4) There is always a relation $\left|g_{i p} g_{j m}\right| \leq\left|g_{i j}\right|$ if $i$ is starred and $j$ is unstarred. 


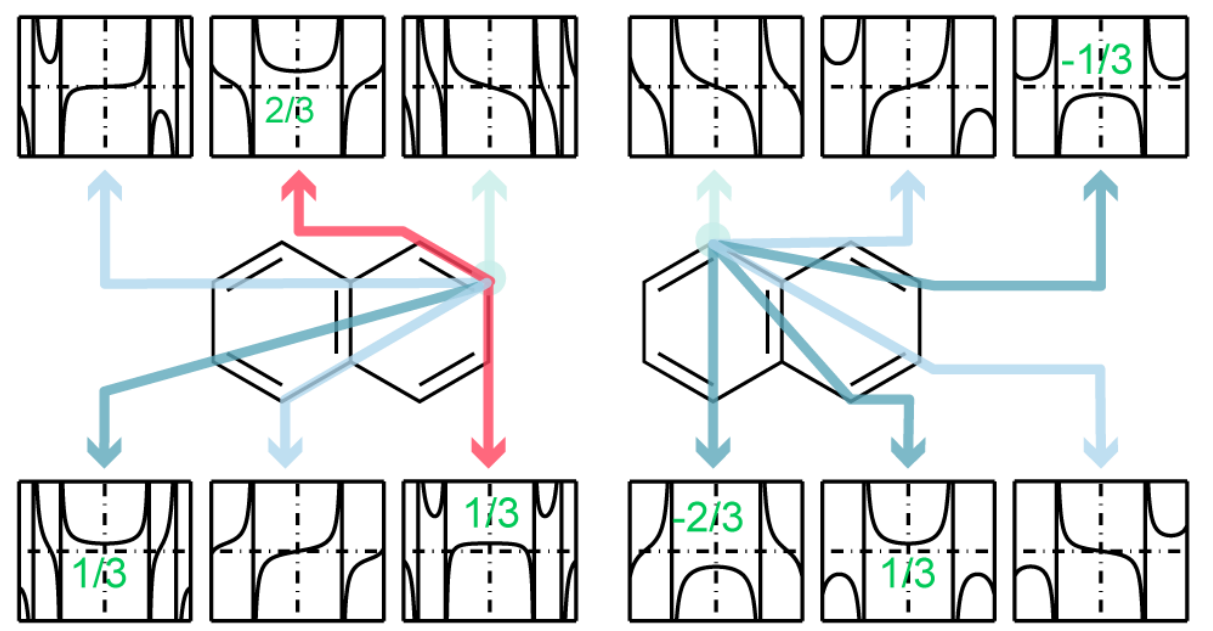

Figure S5. Shape of the zeroth Green functions of typical segments in naphthalene.

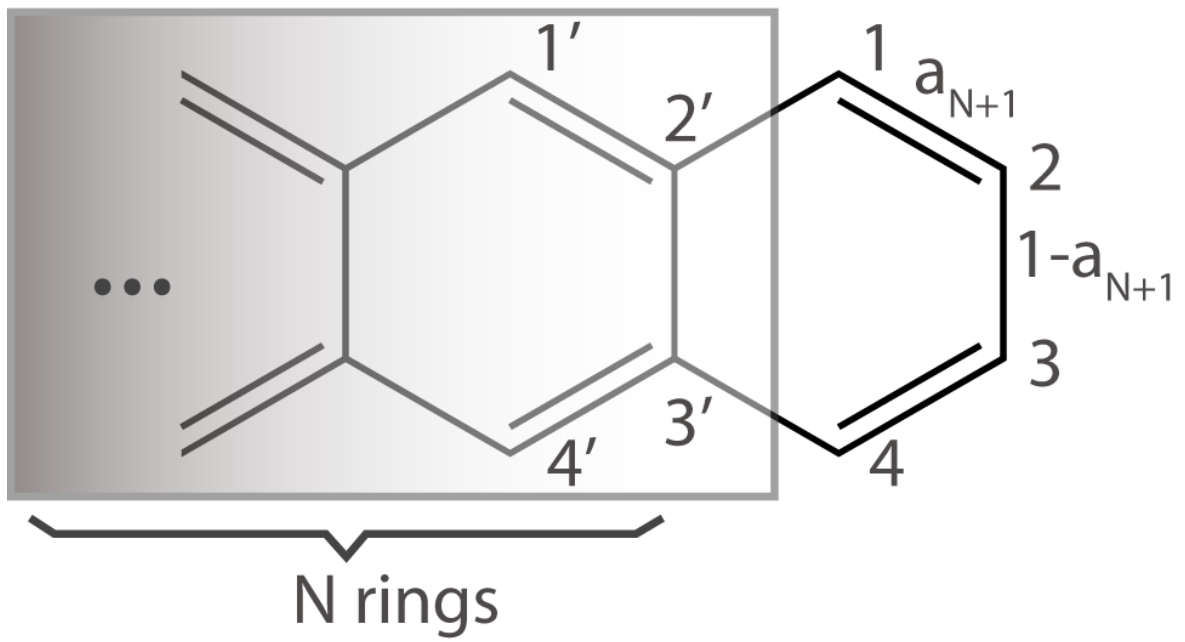

Figure S6. Schmatic of an acene.

Table S 1 Non-zero values of $G^{N+1}{ }_{i 2}, \mathrm{G}^{N+1}{ }_{j 1}, G^{N+1}{ }_{j 3}$ and $G^{N+1}{ }_{i j}$

\begin{tabular}{|c|c|c|c|c|c|}
\hline $\mathrm{i}$ & $\mathrm{j}$ & $G^{N+1}{ }_{i 2}$ & $\mathrm{G}^{N+1}{ }_{j 1}$ & $G^{N+1}{ }_{j 3}$ & $G^{N+1}{ }_{i j}$ \\
\hline 1 & 2 & $a_{N+1}$ & $a_{N+1}$ & $1-a_{N+1}$ & $a_{N+1}$ \\
\hline 1 & 4 & $a_{N+1}$ & $-a_{N+1}$ & $a_{N+1}$ & $-a_{N+1}$ \\
\hline 3 & 2 & $1-a_{N+1}$ & $a_{N+1}$ & $1-a_{N+1}$ & $1-a_{N+1}$ \\
\hline 3 & 4 & $1-a_{N+1}$ & $-a_{N+1}$ & $a_{N+1}$ & $a_{N+1}$ \\
\hline 1 & $\mathrm{j}>4$ & $a_{N+1}$ & $a_{N+1} G_{j^{\prime} 3}^{N}$ & $-a_{N+1} G_{j^{\prime} 3}^{N}$ & $a_{N+1} G_{j^{\prime} 3}^{N}$ \\
\hline 3 & $\mathrm{j}>4$ & $1-a_{N+1}$ & $a_{N+1} G_{j^{\prime} 3}^{N}$ & $-a_{N+1} G_{j^{\prime} 3}^{N}$ & $-a_{N+1} G_{j^{\prime} 3}^{N}$ \\
\hline $\mathrm{i}>4$ & 2 & $-a_{N+1} G_{i^{\prime} 2}^{N}$ & $a_{N+1}$ & $1-a_{N+1}$ & $-a_{N+1} G^{N}{ }^{\prime} 2$ \\
\hline
\end{tabular}




\begin{tabular}{|c|c|c|c|c|c|}
\hline $\mathrm{i}>4$ & 4 & $-a_{N+1} G_{i^{\prime} 2}^{N}$ & $-a_{N+1}$ & $a_{N+1}$ & $a_{N+1} G_{i^{\prime} 2}^{N}$ \\
\hline $\mathrm{i}>4$ & $\mathrm{j}>4$ & $-a_{N+1} G_{i^{\prime} 2}^{N}$ & $a_{N+1} G_{j^{\prime} 3}^{N}$ & $-a_{N+1} G_{j^{\prime} 3}^{N}$ & $G^{N}{ }_{i^{\prime} j^{\prime}}-a_{N+1} G_{i^{\prime} 2}^{N} G_{j^{\prime} 3}^{N}$ \\
\hline
\end{tabular}

\section{Derivation of the segmented analysis}

Below we take the molecule DBT as an example. It contains only one thiophene ring. According to the segmented method introduced in the main text, the carbon part of the Hamiltonian can be divided into two segments, resulting in four blocks $H^{(1)}$, $H^{(2)}, H^{(12)}$ and $H^{(21)}$ as follows:

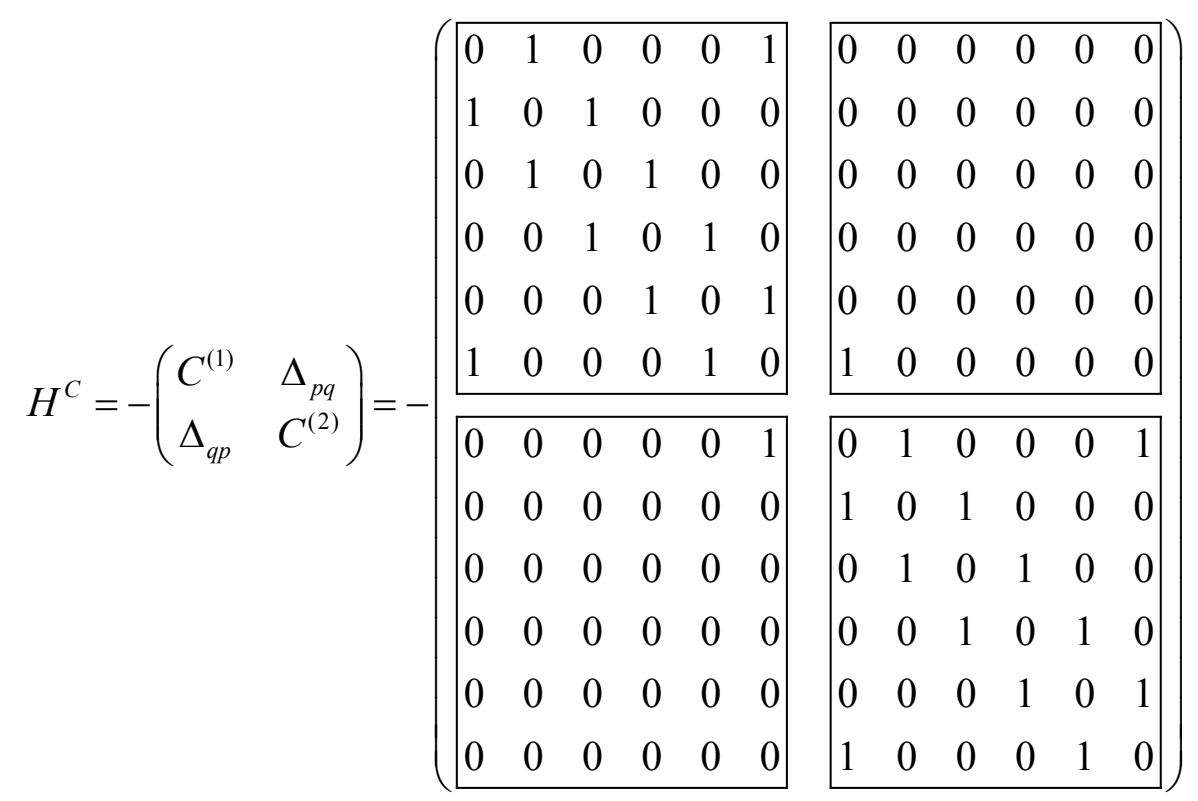

Here $C^{(1) /(2)}$ are the connection matrices of segments (1) and (2) themselves, and $\Delta_{p q}$ denotes an $N_{C} / 2 \times N_{C} / 2$ matrix in which the $(p, q)$ element equals to 1 while all the other elements are zero. The $\mathrm{C}$ atoms numbered by $p$ and $q$ correspond to the connecting atoms between the two segments. On the other hand, the sulphur part of the Hamiltonian is

$$
H^{S}=-h
$$

If there are more than one thiophene rings it will be a unit matrix multiplied by $-h$. At last, the interacting part $H^{S C}$ is

$$
H^{S C}=-k\left(\begin{array}{lllllllllllll}
0 & 0 & 0 & 0 & 1 & 0 & 0 & 1 & 0 & 0 & 0 & 0
\end{array}\right)
$$

In the multi-thiophene case, there will be $N_{S}$ lines each of which have only two non-zero elements on the $m_{u}$ th and $n_{u}$ th column, reflecting the two S-C bonds on each 
$\mathrm{S}$ numbered by $u$. The two $\mathrm{C}$ atoms adjacent to the same $\mathrm{S}$ atom always belong to different segments. Thus Equation (2) can be easily obtained.

Now we begin to analyze the $\mathrm{C}$ backbone segmentally. When it contains only two segments, the fact that $\Delta_{p q}$ has only one non-zero element can be used to simplify the $G^{C}$ function. If we define the Green function of segment $(1) /(2)$ as $\mathrm{g}^{(1) /(2)}$, by direct matrix inversion similar to Equation (1) we obtain

$$
G^{C}(E)=\left(\begin{array}{cc}
G^{(1)}(E) & G^{(12)}(E) \\
G^{(21)}(E) & G^{(2)}(E)
\end{array}\right)
$$

where

$$
\left\{\begin{array}{l}
G^{(1)}(E)=\left[E I-H^{(1)}-g_{q q}^{(2)}(E) \Delta_{p p}\right]^{-1} \\
G^{(2)}(E)=\left[E I-H^{(2)}-g_{p p}^{(1)}(E) \Delta_{q q}\right]^{-1} \\
G_{i j}^{(12)}(E)=-G_{i p}^{(1)}(E) g_{q j}^{(2)}(E) \\
G_{i j}^{(21)}(E)=-G_{i q}^{(2)}(E) g_{p j}^{(1)}(E)
\end{array}\right.
$$

Because $g_{p p}^{(1)}(0)$ and $g^{(2)}{ }_{q q}(0)$ are always zero at $E=0$, the intra-segmental Green function $G^{(1) /(2)}(0)$ is exactly $g^{(1) /(2)}(0)$. Thus the $(i, j)$ element of the inter-segmental Green function is $-g^{(1)}{ }_{\mathrm{i} p}(0) g^{(2)}{ }_{q j}(0)$.

Furthermore, when there are three segments in serial, different blocks of the Green function of the $\mathrm{C}$ backbone has the following form

$$
\left\{\begin{array}{l}
G^{(11)}=\left(E I-H^{(1)}-\left\{\left[E I-H^{(2)}-g_{q_{2} q_{2}}^{(3)} \Delta_{p_{2} p_{2}}\right]^{-1}\right\}\left(q_{1}, q_{1}\right) \Delta_{p_{1} p_{1}}\right)^{-1} \\
G^{(22)}=\left(E I-H^{(2)}-g_{p_{1} p_{1}}^{(1)} \Delta_{q_{1} q_{1}}-g_{q_{2} q_{2}}^{(3)} \Delta_{p_{2} p_{2}}\right)^{-1} \\
G^{(33)}=\left(E I-H^{(3)}-\left\{\left[E I-H^{(2)}-g_{p_{1} p_{1}}^{(1)} \Delta_{q_{1} q_{1}}\right]^{-1}\right\}\left(p_{2}, p_{2}\right) \Delta_{q_{2} q_{2}}\right)^{-1} \\
G_{i j}^{(12)}=-G_{i p_{1}}^{(11)}\left\{\left[E I-H^{(2)}-g_{q_{2} q_{2}}^{(3)} \Delta_{p_{2} p_{2}}\right]^{-1}\right\}\left(q_{1}, j\right) \\
G^{(13)}=-G_{i p_{2}}^{(12)} g_{q_{2} j}^{(3)} \\
G^{(23)}=-G_{i p_{2}}^{(22)} g_{q_{2} j}^{(3)}
\end{array}\right.
$$

Obviously, when there are three or more than three segments in serial, a rule similar with the two-segmental case resists. At $E=0$, the intra-segmental Green function is the same with the Green function of the segment itself. Inter-segmental Green function, on the other hand, become the product of each segment's Green functions on the path multiplied by the factor $(-1)^{x+1}$, where $x$ is the number of Green functions. When $E$ is close to zero, the value of $G^{m o l}(E)$ predicted by the segmental Green functions also serve as a good reference. In Figure S7a-e we draw the $G^{\text {mol }}(E), G^{C}(E)$ 
and $G^{S^{\prime}}(E)$ functions of the connections used in Figure $\mathrm{S} 1$ under the TB approach, and in Figure S8a-e we draw these functions fully predicted by the segmental method. The predicted results serve as good approximations around zero energy.

Then we go back to the simplest two-segmental case. In this case matrices $Z(E)$ and $L(E)$ will reduce to a simple scalar. Assuming $m$ and $p$ belong to (1) and $n$ and $q$ belongs to (2), if $m$ is starred, $q$ is always starred while $n$ and $p$ are unstarred. Thus we take advantage of the easy zeros of starred-starred or unstarred-unstarred connections and obtain

$$
\begin{aligned}
& Z(0)=\frac{1}{2 k^{2}}\left[\frac{h}{2 k^{2}}+g_{m p}^{(1)}(0) g_{n q}^{(2)}(0)\right]^{-1} \\
& L_{i}(0)=G_{i m}^{C}(0)+G_{i n}^{C}(0) \\
& L_{j}(0)=G_{j m}^{C}(0)+G_{j n}^{C}(0)
\end{aligned}
$$

Explicit form of $L_{i}(0)$ depends on which segment $i$ belongs to, where one of $G^{C}{ }_{i m}(0)$ and $G^{C}{ }_{i n}(0)$ must be zero. The final form of $G^{m o l}(0)$ of a two-segmental thienoacene is

$$
G_{i j}^{m o l}(0)=\left\{\begin{array}{c}
g_{i j}^{(1)}+\frac{\left(g_{i m}^{(1)}-g_{i p}^{(1)} g_{n q}^{(2)}\right)\left(g_{j m}^{(1)}-g_{j p}^{(1)} g_{n q}^{(2)}\right)}{2\left(\frac{h}{2 k^{2}}+g_{m p}^{(1)} g_{n q}^{(2)}\right)}, \\
-g_{i p}^{(1)} g_{j q}^{(2)}+\frac{\left(g_{i m}^{(1)}-g_{i p}^{(1)} g_{n q}^{(2)}\right)\left(g_{j n}^{(2)}-g_{j q}^{(2)} g_{m p}^{(1)}\right)}{2\left(\frac{h}{2 k^{2}}+g_{m p}^{(1)} g_{n q}^{(2)}\right)},
\end{array} \quad i \in(1), j \in(2)\right.
$$

The energy dependence of the segmental Green functions is omitted for short. Six detailed cases based on Equation (S21) are summarized in Table 1 in the main text. Using the conclusions of the previous section and taking $h / 2 k^{2}$ as 1 , it is easy to prove $\left|G^{m o l}(0)\right|$ range from $\left|G^{C}(0)\right| / 2$ to $3\left|G^{C}(0)\right| / 2$ for case 1 , and from $3\left|G^{C}(0)\right| / 4$ to $\left|G^{C}(0)\right|$ for case 4 . This is why the S' pathway cannot change the order of the conductance of the $\mathrm{C}$ pathway. The value of $\left|G^{S^{\prime}}(0)\right|$ is always relatively small because the numerator is the product of several multipliers the absolute values of which are less than 1 , and the denominator is always larger than 2. In cases 2 and $3, G^{C}(E)$ is based on an odd segmental Green function which always passes through the origin, so that near the zero energy the S' pathway tend to shift the anti-resonance not far away. In cases 5 and $6, G^{C}(E)$ is based on the product of two segmental Green function while the shape can be predicted according to Figure 2c and Figure S5 for simple molecules. The 
performance of the anti-resonance depends on the specific topology, while the conductance due to the contribution of the S' pathway is also relatively small under the impact of the denominator. Moreover, the shape of the segmental Green functions illustrated in Figure 2a can be used to evaluate the shape of $G^{C}(E)$ without calculation.

We adopt six connections in BDT corresponding to the six cases and draw the precise $G^{m o l}, G^{C}$ and $G^{S^{\prime}}$ functions in Figure S9, along with the segmentally predicted $G^{m o l}$ functions. Similarly, another six connections in the two-segmental DNT are shown in Figure S10. When there are more than two segments, the interactions between $G^{C}$ and $G^{S^{\prime}}$ of some example connections can be found in Figure S7(b, c, e, f) and S11, still obeying rules of the two-segmental case.
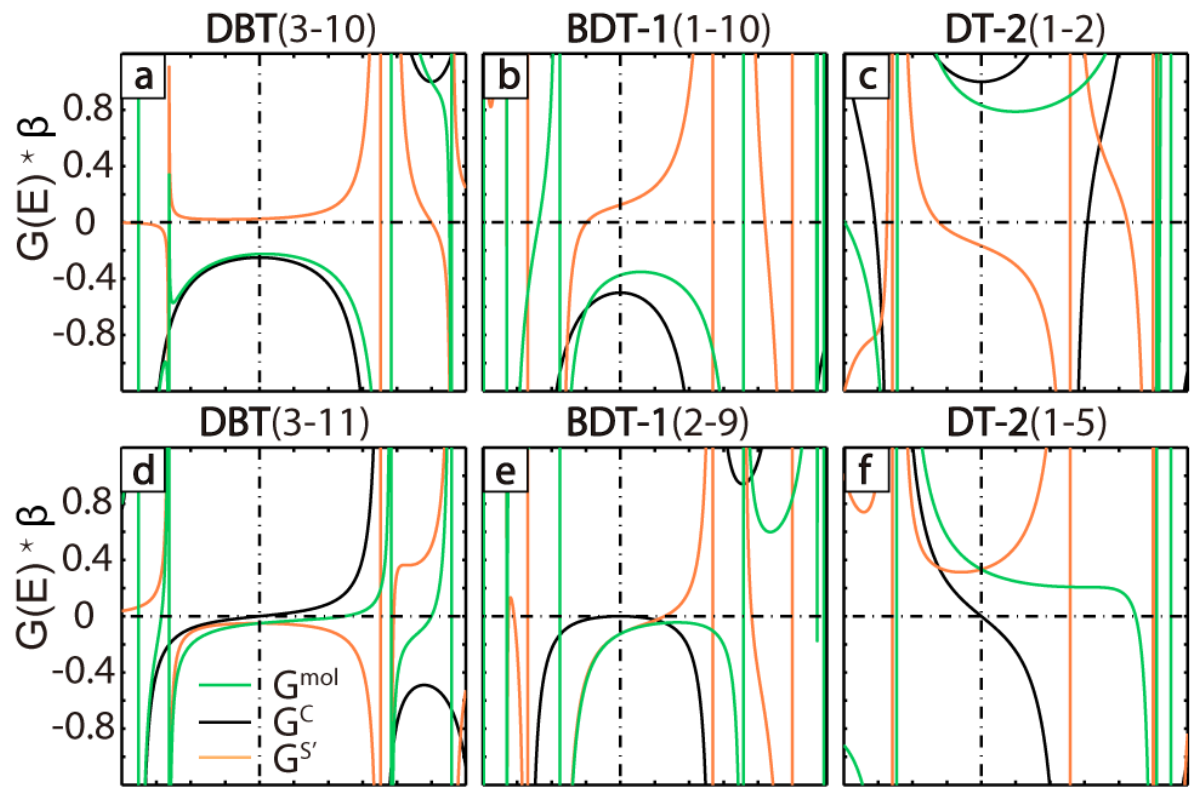

BDT-1(2-9)
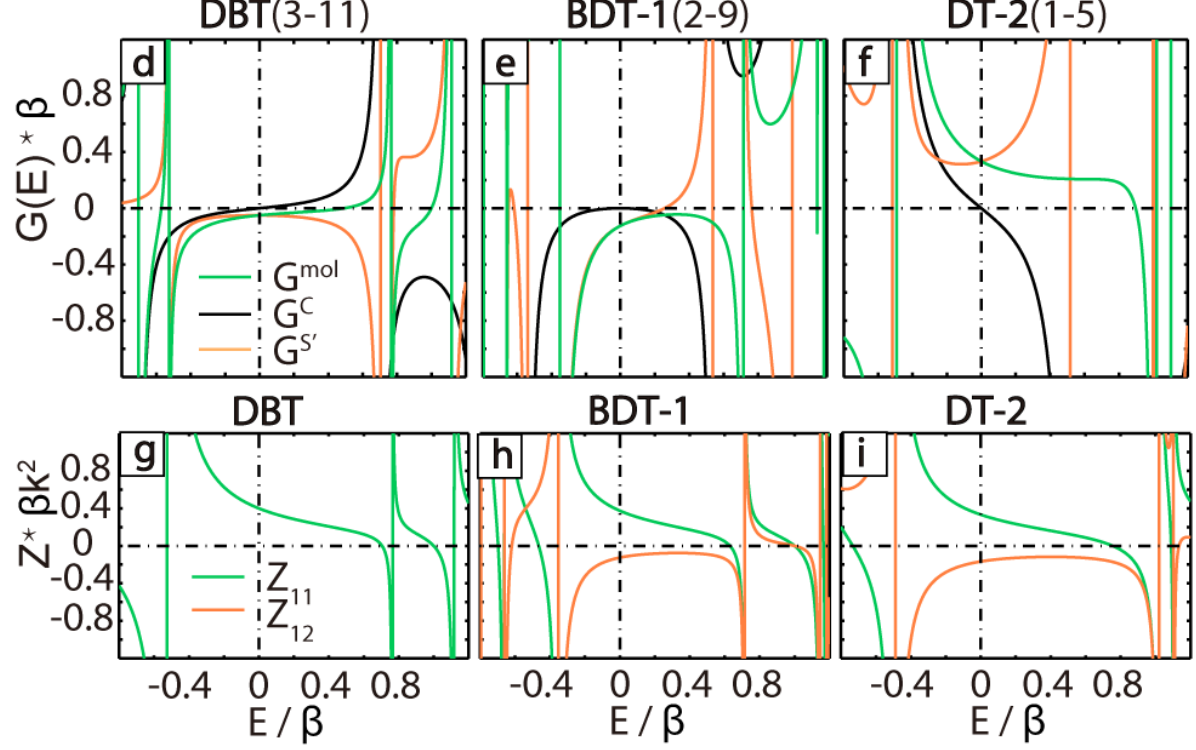

Figure S7. (a-f) $G^{m o l}(E), G^{C}(E)$ and $G^{S^{\prime}}(E)$ of the connections discussed in Figure S1. (g-i) Elements of $Z(E)$ matrix of the molecules DBT, BDT-1 and DT-2. 

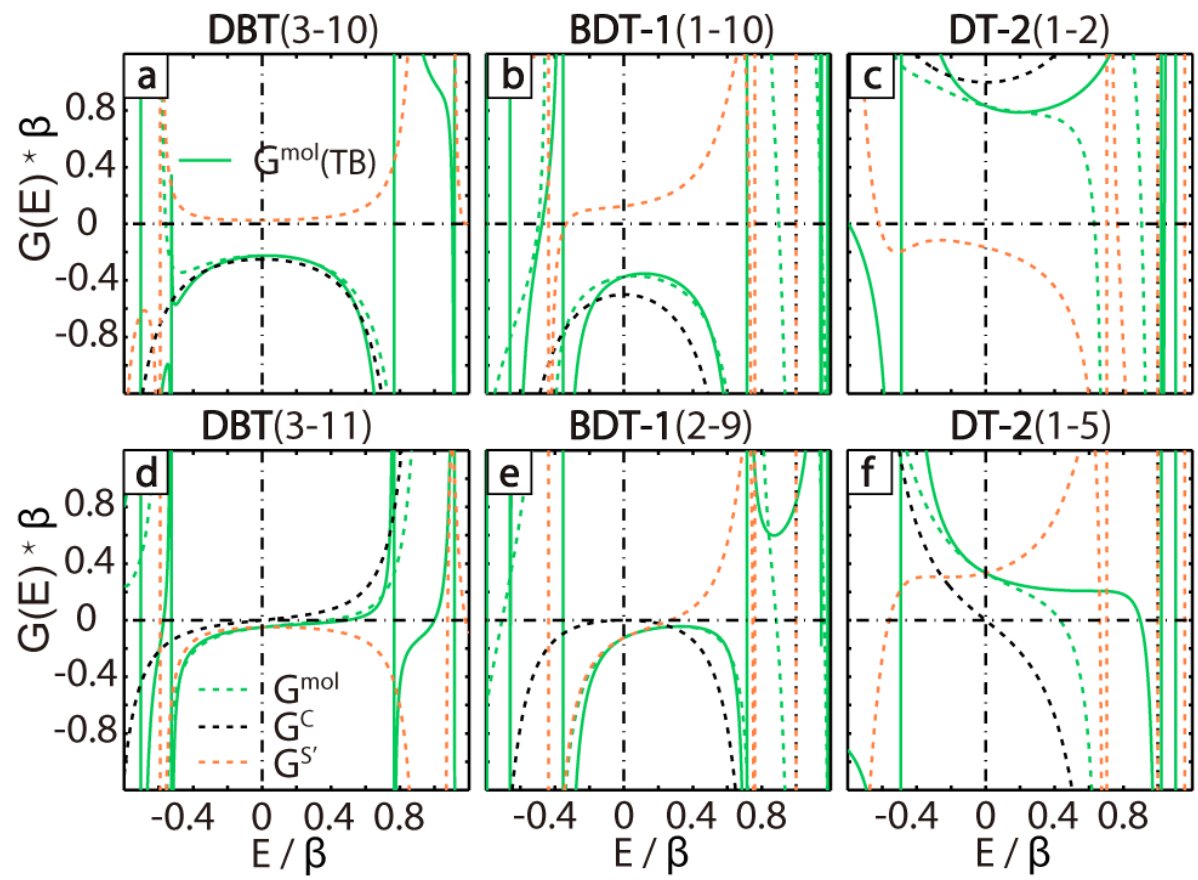

Figure S8. $G^{m o l}(E), G^{C}(E)$ and $G^{S^{\prime}}(E)$ of the connections discussed in Figure S1 predicted by the segmented method. Green solid lines are $G^{m o l}(E)$ functions calculated under the TB approach.
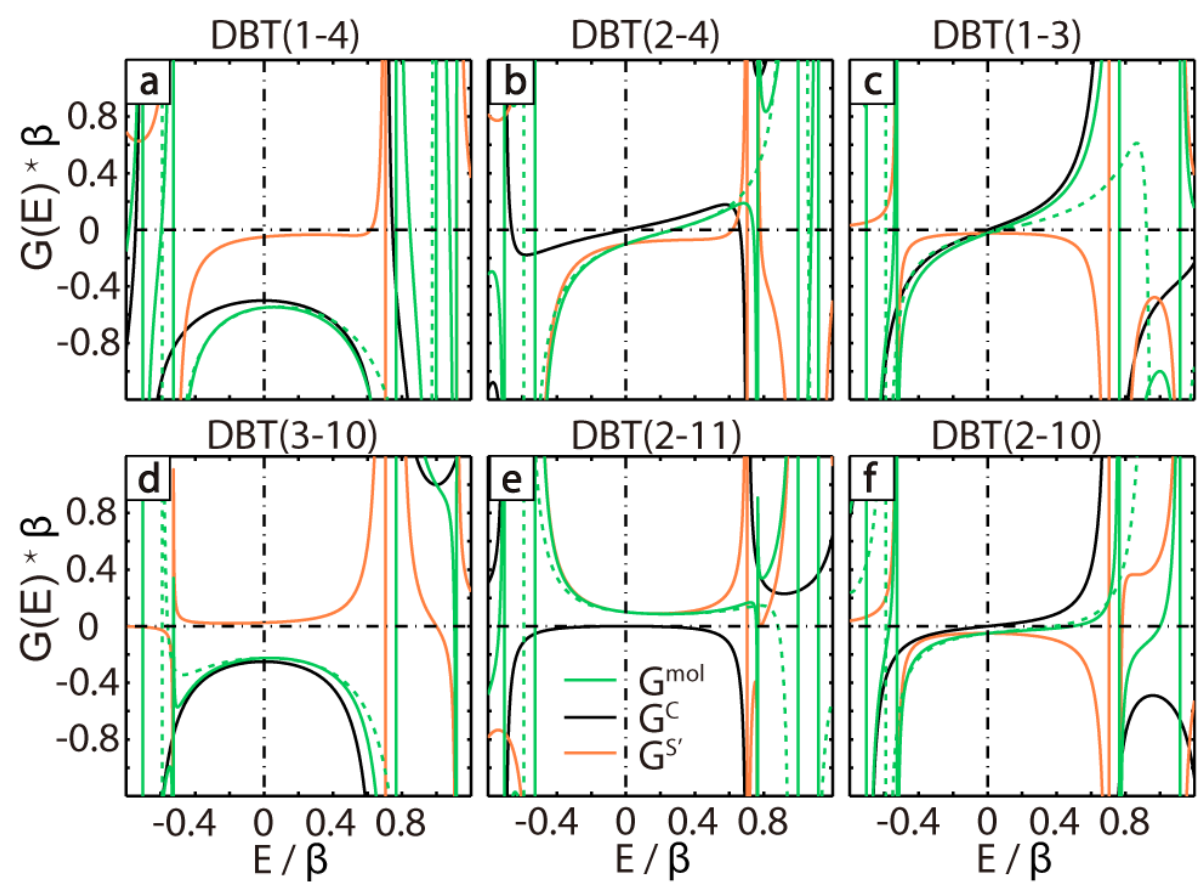

Figure S9. $G^{m o l}(E), G^{C}(E)$ and $G^{S^{\prime}}(E)$ of the six different cases in DBT. Green dashed lines are $G^{m o l}(E)$ predicted by the segmented method. 


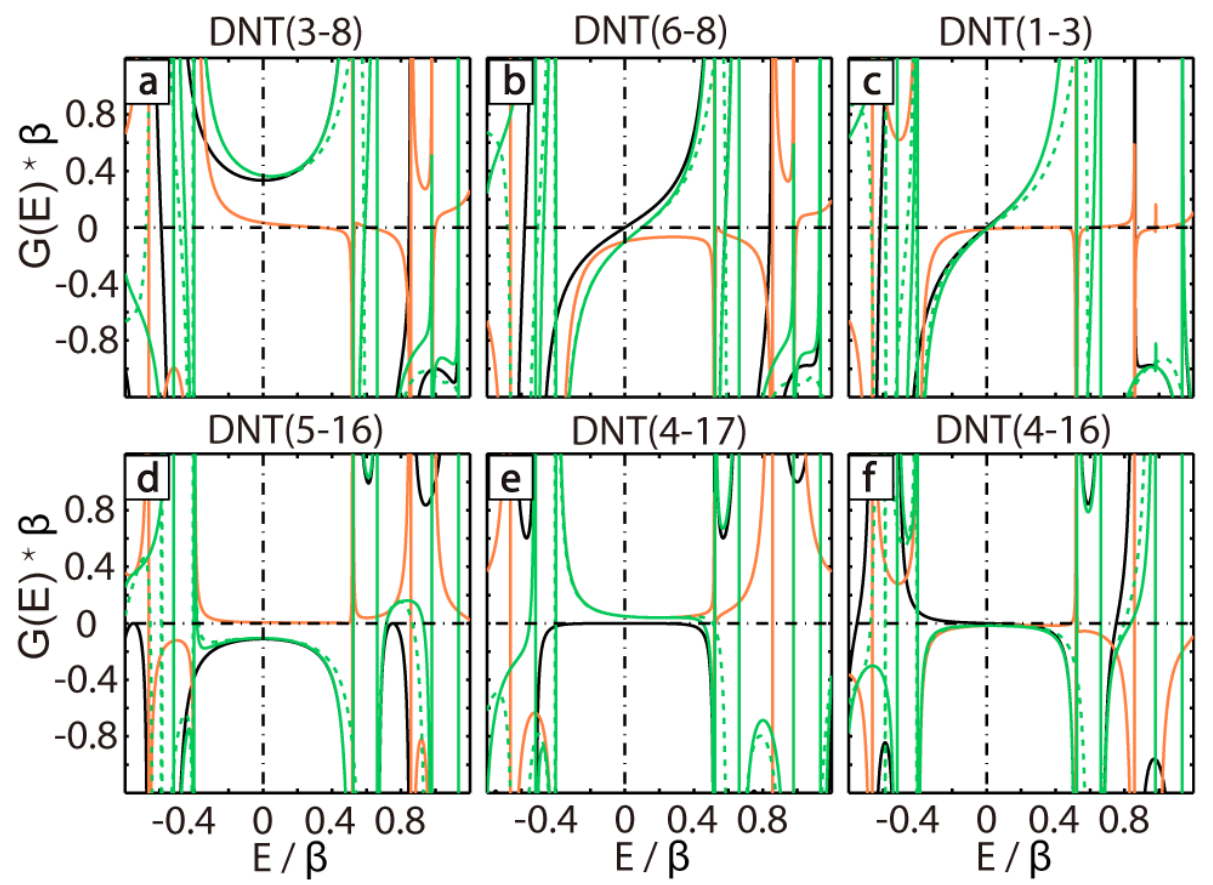

Figure S10. $G^{\text {mol }}(E), G^{C}(E)$ and $G^{S^{\prime}}(E)$ of the six different cases in DNT. Green dashed lines are $G^{\text {mol }}(E)$ predicted by the segmented method.
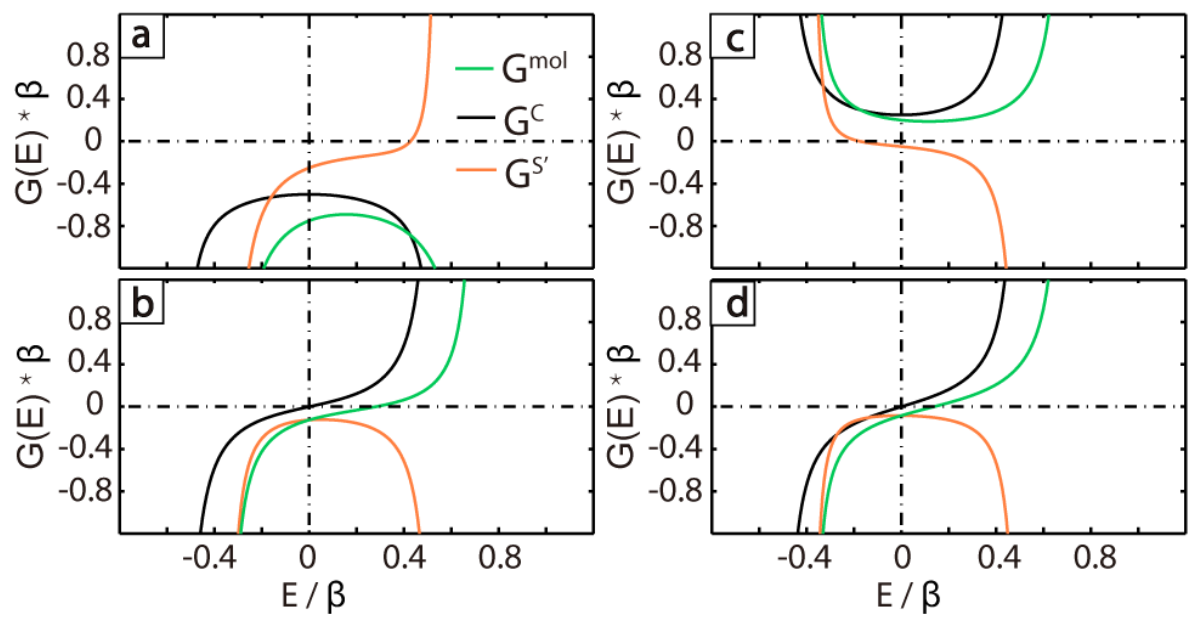

Figure S11. $G^{m o l}(E)$ of the four connections shown in Figure 1b-c and their two terms decomposed onto the two pathways between the HOMO and LUMO resonances. 


\section{Intra-segmental and inter-segmental Green functions}
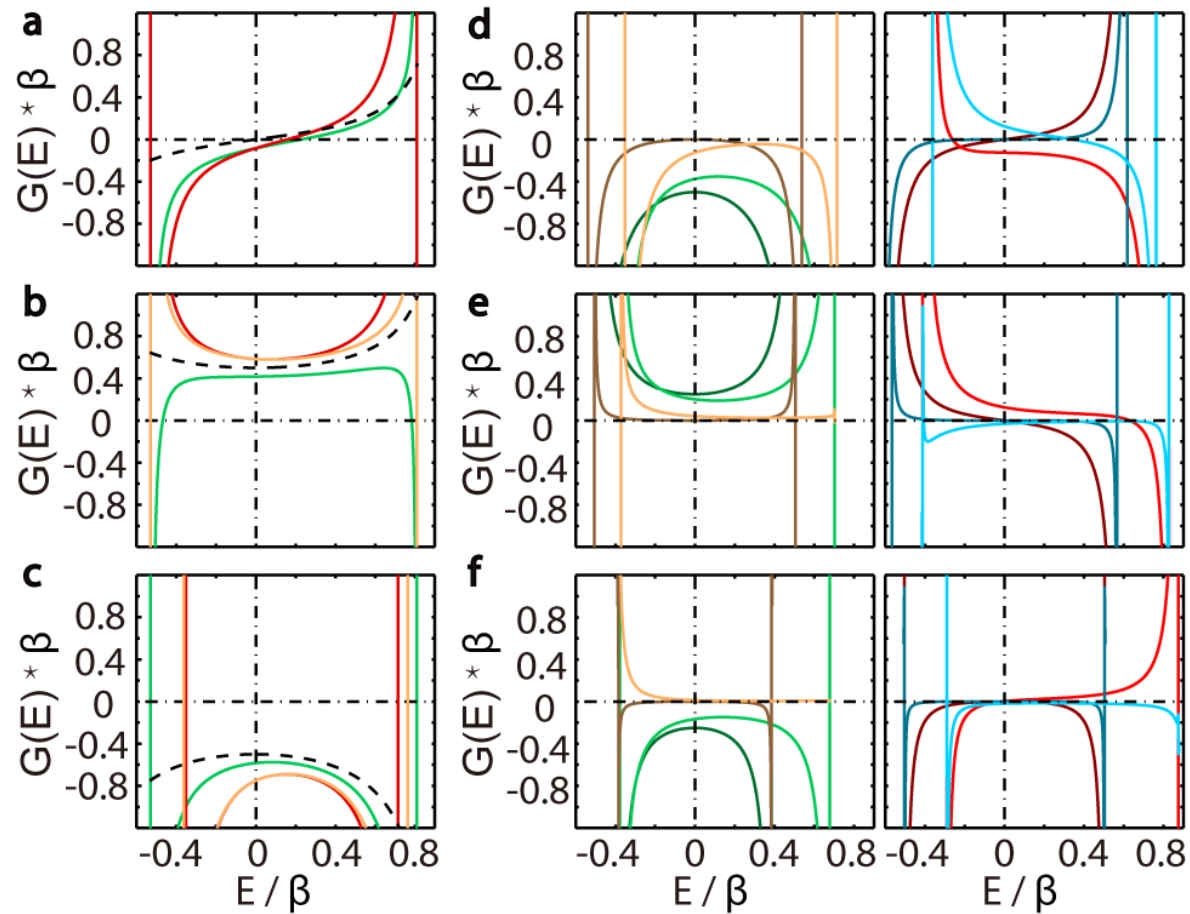

Figure S12. Green functions between HOMO and LUMO resonances under the TB approach corresponding to the connections in Figure 3 (a-c) and 4 (d-f). The darker solid lines correspond to the contribution of $G^{C}$.

\section{Atomic projected density of states (PDOS) analysis}

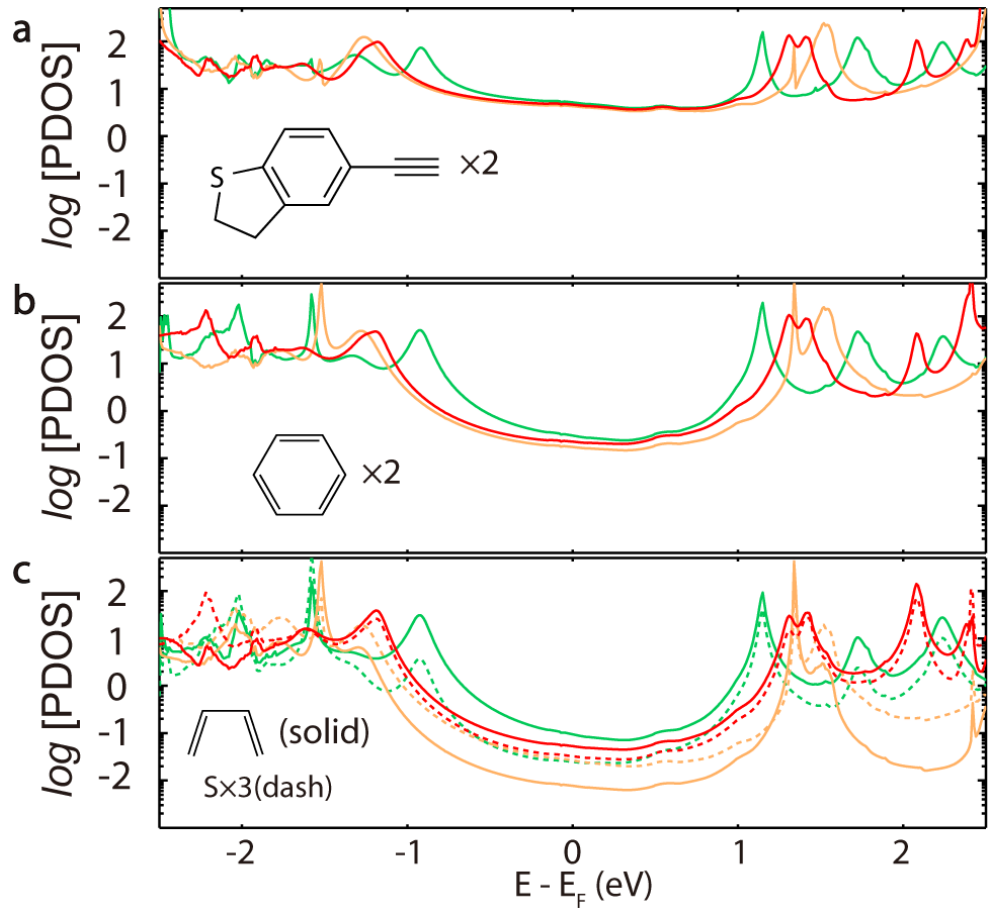

Figure S13. Projected DOS of different parts of the connections in Figure 4g. 
It should be noted here in the NEGF+DFT calculations, the calculated Fermi levels of the $1 \mathrm{D}$ and 3D electrodes are $-5.401 \mathrm{eV}$ and $-4.412 \mathrm{eV}$ respectively using SZP basis set. The vacuum levels are obtained by slab model with vacuum height more than $14 \AA$. For 1D Au electrode we use 7 atoms, the same with the length of the electrode on one side of the transport model. For 3D Au electrode we use $10 \mathrm{Au}(111)$ layers according to the whole transport model as it is periodic in the transport direction.

\section{References}

1. Yoshizawa, K. An Orbital Rule for Electron Transport in Molecules. Acc. Chem. Res. 2012, $45,1612-1621$.

2. Geng, Y.; Sangtarash, S.; Huang, C.; Sadeghi, H.; Fu, Y.; Hong, W.; Wandlowski, T.; Decurtins, S.; Lambert, C. J.; Liu, S. X. Magic ratios for connectivity-driven electrical conductance of graphene-like molecules. J. Am. Chem. Soc. 2015, 137, 4469-4476.

3. Liu, X.; Sangtarash, S.; Reber, D.; Zhang, D.; Sadeghi, H.; Shi, J.; Xiao, Z. Y.; Hong, W.; Lambert, C. J.; Liu, S. X. Gating of Quantum Interference in Molecular Junctions by Heteroatom Substitution. Angew. Chem. Int. Ed. 2017, 56, 173-176.

4. Borges, A.; Solomon, G. C. Effects of Aromaticity and Connectivity on the Conductance of Five-Membered Rings. J. Phys. Chem. C 2017, 121, 8272-8279.

5. Takimiya, K.; Shinamura, S.; Osaka, I.; Miyazaki, E. Thienoacene-based organic semiconductors. Adv. Mater. 2011, 23, 4347-4370.

6. Sangtarash, S.; Huang, C.; Sadeghi, H.; Sorohhov, G.; Hauser, J.; Wandlowski, T.; Hong, W.; Decurtins, S.; Liu, S. X.; Lambert, C. J. Searching the Hearts of Graphene-like Molecules for Simplicity, Sensitivity, and Logic. J. Am. Chem. Soc. 2015, 137, 11425-11431.

7. Tsuji, Y.; Hoffmann, R.; Movassagh, R.; Datta, S. Quantum interference in polyenes. J. Chem. Phys. 2014, 141, 224311. 